\title{
Discovery of a bipolar X-ray jet from the T Tauri star DG Tauri
}

\author{
M. Güdel ${ }^{1,2,3}$, S. L. Skinner ${ }^{4}$, M. Audard ${ }^{5,6}$, K. R. Briggs ${ }^{1}$, and S. Cabrit ${ }^{7}$ \\ 1 Paul Scherrer Institut, Würenlingen and Villigen, 5232 Villigen PSI, Switzerland \\ e-mail: [guedel; briggs]@astro.phys.ethz.ch \\ 2 Max-Planck-Institute for Astronomy, Königstuhl 17, 69117 Heidelberg, Germany \\ 3 Leiden Observatory, Leiden University, PO Box 9513, 2300 RA Leiden, The Netherlands \\ ${ }^{4}$ CASA, 389 UCB, University of Colorado, Boulder, CO 80309-0389, USA \\ e-mail: skinners@casa.colorado.edu \\ 5 Integral Science Data Centre, Ch. d'Écogia 16, 1290 Versoix, Switzerland \\ e-mail: marc . audard@obs . unige.ch \\ ${ }^{6}$ Observatoire de Genève, University of Geneva, Ch. de Maillettes 51, 1290 Sauverny, Switzerland \\ 7 LERMA, Observatoire de Paris, UMR 8112 CNRS, 61 avenue de l'Observatoire, 75014 Paris, France \\ e-mail: sylvie.cabrit@obspm.fr
}

Received 22 June 2007 / Accepted 6 December 2007

\section{ABSTRACT}

\begin{abstract}
Aims. We have obtained and analyzed Chandra ACIS-S observations of the strongly accreting classical T Tauri star DG Tau. Our principal goals are to map the immediate environment of the star to characterize possible extended X-rays formed in the jet, and to re-visit the anomalous, doubly absorbed X-ray spectrum of DG Tau itself.

Methods. We combine our new ACIS-S data with a data set obtained previously. The data are superimposed to obtain flux and hardness images. Separate X-ray spectra are extracted for DG Tau and areas outside its point spread function.

Results. We detect a prominent X-ray jet at a position angle of PA $\approx 225$ deg (tentatively suggested by Güdel et al. 2005, ApJ, 626, L53), coincident with the optical jet axis. We also identify a counter jet at PA $=45 \mathrm{deg}$. The X-ray jets are detected out to a distance of $\approx 5^{\prime \prime}$ from the star, their sources being extended at the ACIS-S resolution. The jet spectra are soft, with a best-fit electron temperature of $3.4 \mathrm{MK}$. We find evidence for excess absorption of the counter jet. The spectrum of the DG Tau point source shows two components with largely different temperatures and absorption column densities.

Conclusions. The similar temperatures and small absorbing gas columns of the jet sources and the soft component of the "stellar" source suggest that these sources are related, produced either by shocks or by magnetic heating in the jets. Cooling estimates suggest that the pressure in the hot gas contributes to jet expansion. The hard "stellar" component, on the other hand, is associated with a stellar corona or magnetosphere. The excessive photoelectric absorption of this component suggests the presence of dust-depleted accretion streams above coronal magnetic fields.
\end{abstract}

Key words. stars: coronae - stars: formation - stars: individual: DG Tau - stars: pre-main sequence - stars: winds, outflows X-rays: stars

\section{Introduction}

Pre-main sequence stars show various signs of accretion and outflow, such as stellar winds inducing high mass-loss rates (e.g., Dupree et al. 2005; Johns-Krull \& Herczeg 2007; Kwan et al. 2007), molecular outflows observed in molecular lines (e.g., Bachiller 1996), and accompanying optical (e.g., Hirth et al. 1997; Eislöffel \& Mundt 1998) and radio jets (e.g., Anglada 1995). The most evident manifestation of outflows are the optically visible jets and their associated Herbig-Haro $(\mathrm{HH})$ objects at distances up to several arcminutes from the star. These structures are excited by internal shocks or in regions where the fast mass stream encounters the interstellar medium and shock-ionizes the gas (for a review of Herbig-Haro flows, see Reipurth \& Bally 2001). Under ideal circumstances (low extinction, strong ionization), optical jets can be identified at distances as close as $0{ }^{\prime} 1$ to the star (Bacciotti et al. 2002). The same compact jets are also routinely detected at radio wavelengths, where the emission mechanism is thought to be bremsstrahlung from the shock-heated gas (Rodríguez 1995; Anglada 1995). Radio brightness temperatures suggest overall gas temperatures of order $10^{4} \mathrm{~K}$. This picture is ambiguous, however, as a number of non-thermal jets have been suggested from radio polarization or synchrotron-like spectral shapes (e.g., Curiel et al. 1993; Ray et al. 1997). Magnetic fields may thus play a role not only in launching the jets, but in their propagation as well.

Outflow processes are prone to producing X-rays, given that shocks with shock jump velocities of order several hundred $\mathrm{km} \mathrm{s}^{-1}$ are possible. The relevant theory and a simple model have been discussed by Raga et al. (2002). The strongshock temperature can be expressed as $T \approx 1.5 \times 10^{5} v_{100}^{2} \mathrm{~K}$ (for fully ionized gas) where $v_{100}$ is the shock speed relative to a target in units of $100 \mathrm{~km} \mathrm{~s}^{-1}$. Jet speeds are typically of order $v=300-500 \mathrm{~km} \mathrm{~s}^{-1}$ (Eislöffel \& Mundt 1998; Anglada 1995; Bally et al. 2003), in principle allowing for shock speeds of similar magnitude. If a flow shocks a standing medium at $400 \mathrm{~km} \mathrm{~s}^{-1}$, then $T \approx 2.4 \mathrm{MK}$.

Faint, soft X-ray emission has been detected from a few protostellar HH objects (Pravdo et al. 2001, 2004; Pravdo \& Tsuboi 2005; Favata et al. 2002; Bally et al. 2003; Tsujimoto et al. 2004; Grosso et al. 2006). Bally et al. (2003) used a Chandra observation to show that X-rays form within an arcsecond of the 
Table 1. Observations.

\begin{tabular}{|c|c|c|c|}
\hline \multicolumn{4}{|c|}{ Previous observations: } \\
\hline Instrument & Chandra ACIS-S & XMM-Newton EPIC & \\
\hline ObsID & 4487 & 0203540201 & \\
\hline Start time (UT) & 2004-01-11 02:58:51 & 2004-08-17 06:08:10 & \\
\hline End time (UT) & 2004-01-11 11:52:21 & 2004-08-17 17:32:46 & \\
\hline Exposure time (s) & 29717 & 41076 & \\
\hline \multicolumn{4}{|l|}{ New observations: } \\
\hline Instrument & Chandra ACIS-S & Chandra ACIS-S & Chandra ACIS-S \\
\hline ObsID & 6409 & 7247 & 7246 \\
\hline Start time (UT) & 2005-12-15 11:03:14 & 2005-12-17 09:37:05 & 2006-04-12 17:13:23 \\
\hline End time (UT) & $2005-12-15$ 16:17:01 & 2005-12-17 14:34:37 & 2006-04-13 01:37:19 \\
\hline Exposure time (s) & 16252 & 15946 & 27811 \\
\hline
\end{tabular}

protostar L1551 IRS-5 while the star itself is too heavily obscured to be detected. As this example illustrates, the jetlaunching region of powerful protostellar jets is often inaccessible to optical, near-infrared or X-ray studies due to excessive absorption. However, a class of strongly accreting, optically revealed classical T Tauri stars (CTTS) also exhibits so-called micro-jets visible in optical lines (Hirth et al. 1997), with flow speeds similar to protostellar jets. CTTS micro-jets have the unique advantage that they can - in principle - be followed down to the acceleration region close to the star both in the optical and in X-rays. For example, Bacciotti et al. $(2000,2002)$ used the HST to trace the jet of the CTTS DG Tau to within 0.' 1 of the star.

DG Tau is a most outstanding T Tauri star for X-ray studies. A Chandra high-resolution X-ray image has shown tentative evidence for the presence of faint X-rays along the optical jet. Both XMM-Newton (Güdel et al. 2007b) and Chandra (Güdel et al. 2005) low-resolution CCD spectra of DG Tau are anomalous, showing a "two-absorber X-ray" (TAX) spectrum in which two independent X-ray components are each subject to different absorption column densities.

To study DG Tau's X-ray emission further, we obtained new Chandra observations that, together with the previous observations, result in three times more Chandra exposure than analyzed before. The present paper describes the new observations, puts them into a context of the previous results, and discusses some tentative models for the jet X-ray emission.

\section{The target: DG Tau and its jets}

\subsection{An accreting, jet-driving T Tauri star}

DG Tau is a single classical T Tau star (Leinert et al. 1991), surrounded by a disk of dust (Dutrey et al. 1996; Testi et al. 2002) and gas (Dutrey et al. 1996; Kitamura et al. 1996a,b = K96a,b; Testi et al. 2002). The dust disk as detected in the millimeter continuum is relatively compact (due to limited sensitivity of present-day instrumentation to optically thin emission of the outer disk), with a size of $11^{\prime \prime} 1 \times 0$.' 6 (Dutrey et al. 1996, also $\mathrm{K} 96 \mathrm{~b}$ ). Dust is also present at larger distances as inferred from extinction of the counter jet (see below). CO gas remains easier to detect at large distances from the star; K96a,b find a ${ }^{13} \mathrm{CO}$ disk size of about $40^{\prime \prime} \times 30^{\prime \prime}$ although we note that this structure is not a Keplerian disk but more akin to a residual, flattened envelope. Interestingly, line shifts as a function of position indicate that the outer parts of the disk are expanding, perhaps driven by interactions between the stellar wind and the disk surface. This latter wind would also be responsible for blowing off residual envelope gas; a spherical gas envelope is indeed not present anymore, as inferred from modeling of the ${ }^{13} \mathrm{CO}$ line (K96a).

DG Tau is a strongly accreting CTTS, with an observed accretion rate of $\dot{M}=\left(10^{-7.34}-10^{-6.13}\right) M_{\odot} \mathrm{yr}^{-1}$ (White \& Ghez 2001; White \& Hillenbrand 2004). It also ejects a well studied collimated jet with knots and bow shocks out to at least $11^{\prime \prime}$, with velocities of several $100 \mathrm{~km} \mathrm{~s}^{-1}$ (e.g., Lavalley et al. 1997; Eislöffel \& Mundt 1998; Dougados et al. 2000). Optical evidence for a counter jet has been reported (Lavalley et al. 1997; Pyo et al. 2003).

\subsection{Previous $X$-ray observations}

We have previously obtained two short X-ray observations of DG Tau (Table 1). Our previous Chandra ACIS-S observation (Güdel et al. 2005) showed tentative indications of very faint, soft emission along the optical forward jet out to a distance of about $5^{\prime \prime}$ to the SW. A total of 17 excess counts were collected (including the area around the faint counter jet) in the energy range of $0.4-2.4 \mathrm{keV}$.

The X-ray spectrum of DG Tau itself revealed two independent X-ray components in both the Chandra and XMM-Newton observations (Güdel et al. 2005, 2007b); a soft, little absorbed component is emitted by cool $(\approx 3-4 \mathrm{MK})$ plasma; and a hard, strongly absorbed component originates from hot, occasionally flaring ( $\approx 20-70 \mathrm{MK}$ ) plasma. The soft component was attributed to emission from the base of the jets.

\section{New Chandra observations}

We have obtained new observations of DG Tau with Chandra ACIS-S (Table 1), for a total of $\approx 60 \mathrm{ks}$ of exposure time. We have merged these data with the previously obtained Chandra data to produce images with an equivalent exposure time of approximately $90 \mathrm{ks}$, i.e., three times as much as reported in Güdel et al. (2005). The new observations were collected in three segments, two shorter ones in December 2005 (ObsID 6409 and 7247) and a longer one in April 2006 (ObsID 7246). The data reduction followed standard Chandra CIAO analysis threads ${ }^{1}$ as described in Güdel et al. (2005). We used the "Very Faint" mode to efficiently reduce background radiation. DG Tau's optical sky coordinates for Epoch 2005.0 are $\mathrm{RA}(2000.0)=04^{\mathrm{h}} 27^{\mathrm{m}} 04.697^{\mathrm{s}}$, $\operatorname{Dec}(2000.0)=+26^{\circ} 06^{\prime} 16^{\prime \prime} 10$ (Ducourant et al. 2005).

We extracted stellar X-ray spectra individually for each observation using counts within $11^{\prime \prime} 4-1$ '.7 (depending on the shape

\footnotetext{
1 http://cxc.harvard.edu/ciao/guides/acis_data.html; CIAO version 3.3.0.1 was used.
} 

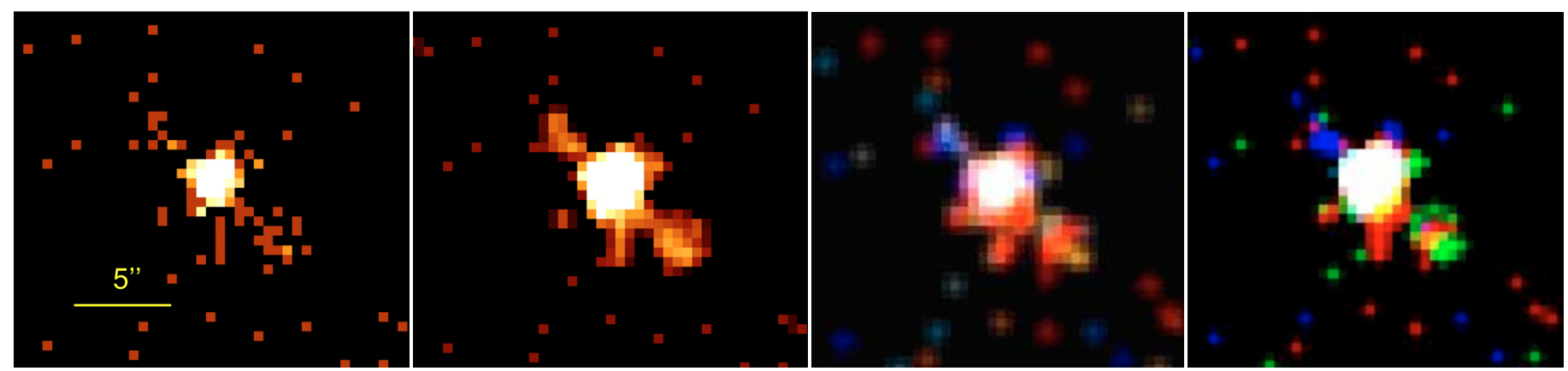

Fig. 1. Chandra ACIS-S images of DG Tau and its jets. North is up and east is to the left. From left to right: a) count image for the 0.6-1.7 keV range; b) same but smoothed; c) color-coded, smoothed hardness image; d) same but with different color coding (see text for details). Pixel size is 0.492 .

of the stellar image) of the centroid position of the DG Tau stellar source. This extraction radius contains approximately $95 \%$ of the power in the PSF (see Chandra Proposers' Observatory Guide [POG] v.9 $)^{2}$. Counts from regions attributed to the jets were extracted from circles with radii of $1.75^{\prime \prime}$ and $2.6^{\prime \prime}$, offset by a few arcseconds to the NE and SW, respectively (see Güdel et al. 2005, for an illustration).

Spectra were produced for DG Tau, the SW (forward) jet, and the NE (counter) jet for each observation (using the CIAO specextract task). We fitted the spectra with simple thermal, collisional-ionization equilibrium models (vapec) in the XSPEC software package (version 11.3.1; Arnaud 1996), complemented with photoelectric absorption models. DG Tau required two thermal models that were each subject to different amounts of absorption. The two jet sources were fitted jointly (see below). We fixed all element abundances at values that are typical for $\mathrm{T}$ Tauri stars, as used in the XEST project (Güdel et al. 2007a; Telleschi et al. 2007a, and references therein $)^{3}$.

Because the ACIS-S background level is negligible for our small sources (see below), we took advantage of the C statistic (Cash 1979) for the spectral fits in XSPEC, applied to unbinned spectra in the energy range of $0.2-7 \mathrm{keV}$ for DG Tau and $0.2-2.2 \mathrm{keV}$ for the jet sources.

\section{The X-ray jets of DG Tau}

Our X-ray images (Fig. 1) provide clear evidence for both a forward (to the SW) and a counter-jet (to the NE), symmetrically arranged outside the stellar point-spread function (PSF) out to distances of about $5^{\prime \prime}$. This is the first and so far only youngstellar double X-ray jet that can be followed essentially down to the star. We now discuss the spatial and spectral results in detail.

\subsection{Jet morphology}

An X-ray image of the DG Tau environment is shown in Fig. 1a, with a pixel size of 0 . $^{\prime} 492$. This image was produced by combining counts from all four Chandra observations. The jet proper motion amounts to about 0.'15-0.'3 per year (Eislöffel \& Mundt 1998 for the 1983-1990 time interval; Dougados et al. 2000 for 1994-1997). If the proper motion was similar during the time interval of our Chandra observations (2004-2006), then the jet

\footnotetext{
2 http://cxc.harvard.edu/proposer/POG/html/

3 The adopted abundances are, with respect to the solar photospheric abundances given by Anders \& Grevesse (1989): $\mathrm{C}=0.45$, $\mathrm{N}=0.788, \mathrm{O}=0.426, \mathrm{Ne}=0.832, \mathrm{Mg}=0.263, \mathrm{Al}=0.5, \mathrm{Si}=0.309$, $\mathrm{S}=0.417, \mathrm{Ar}=0.55, \mathrm{Ca}=0.195, \mathrm{Fe}=0.195, \mathrm{Ni}=0.195$.
}

features would shift by about one ACIS-S pixel between the earliest and the latest observation; such a shift is not critical given the size of the jet source (see below). To minimize the extension of the combined image of the DG Tau stellar PSF due to slight systematic offsets between the attitude solutions of the observations, we determined the stellar centroid coordinates in each exposure using the CIAO wavdetect task, and then shifted the centroids to a common coordinate (the maximum shift applied to one of the exposures was $0 . ' 42$, or less than one pixel). Also, the standard "pixel randomization" procedure was turned off (but was left on for the data sets used for spectral extraction). Because the boresight coordinates are slightly different for the different exposures, the data were reprojected to a common tangent point, using the reproject_events task in CIAO.

To suppress background and to emphasize the soft jet sources, only counts falling within the $0.6-1.7 \mathrm{keV}$ range are plotted in the figure. There is clear evidence for a jet-like extension to the SW along a position angle of $\approx 225 \mathrm{deg}$, but we also find a significant excess of counts in the NE direction $(\mathrm{PA} \approx 45 \mathrm{deg}$ ). This is coincident with the jet optical axis, which for the SW jet has been given as 222 deg (Lavalley et al. 1997), 226 deg (Solf \& Böhm 1993), or, depending on individual knots, 217-237 deg (Eislöffel \& Mundt 1998). We are not aware of any background sources in this region that could produce additional $\mathrm{X}$-ray emission.

Only very few counts from the jet regions fall outside the $\approx 0.6-1.7 \mathrm{keV}$ energy range; we therefore use this range for our statistics (somewhat different from the energy range $0.4-2.4 \mathrm{keV}$ used by Güdel et al. 2005). The SW jet contains, within the extraction circle defined here, 7 and 11 counts for the 2004 and the combined 2005/06 observations, respectively (including one count in the SW jet at $0.58 \mathrm{keV}$ in ObsID 6409). For the NE jet, the numbers are 4 and 5 counts, respectively. Based on the 2004 observation, we would have expected to detect, in the 2005/06 observations, a total of 14 counts in the SW jet and 8 counts in the NE jet, but the differences to the actually observed counts are within about $1 \sigma$ of the uncertainties due to counting statistics, and are therefore not significant. The combined Chandra exposures thus collected a total of 18 and 9 counts in the SW and the $\mathrm{NE}$ jet, respectively (the nearest counts outside this energy range were: one count at $0.43 \mathrm{keV}$ in the SW jet of ObsID 4487; and one count at $2.1 \mathrm{keV}$ in the SW jet of ObsID 6409).

We then estimated potential contamination from the diffuse (sky and detector) background and from the PSF of the bright stellar DG Tau X-ray source. The diffuse background was estimated by extracting counts from a large area in the vicinity of the DG Tau system and scaling them to the jet extraction areas. We found the background contribution to be, statistically, 0.6 and 0.3 cts for the SW and the NE jet area, respectively. We modeled 

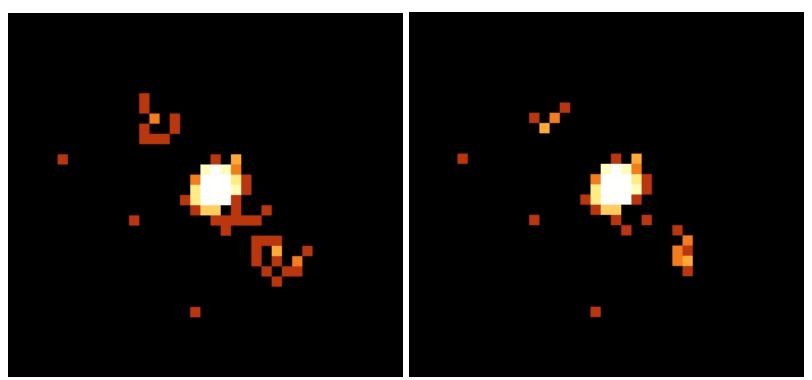

Fig. 2. Comparison of two simulations of the DG Tau X-ray sources. Left: the two jet sources are extended; the NE jet source is represented by a Gaussian source with sigma $=0 . ' 8$; the SW jet source consists of a Gaussian with sigma $=1^{\prime \prime}$ plus a linear source at a position angle of 225 deg between star and Gaussian source. The number of counts in each source is similar to the true observations for an exposure time of $90 \mathrm{ks}$. Only counts in the range of $0.6-1.7 \mathrm{keV}$ are shown. Right: Similar, but both jet sources have been defined as point sources. Pixel size is $0 !^{\prime} 492$.

the DG Tau PSF using the MARX software ${ }^{4}$. Boresight coordinates and roll angles were identical to those of the observations, and the simulated source was put at the sky coordinates of DG Tau (the simulations were done separately for the 2004 and the 2005/06 observations, but the results agreed with each other). The simulation used the best-fit spectrum of DG Tau except that the flux (or the exposure time) was much higher. We then extracted counts in the PSF wings using the identical extraction regions used for the jets, in the $0.6-1.7 \mathrm{keV}$ range, and found a statistical contribution of 0.9 and 0.7 cts of the PSF wings to the SW and NE jet source, respectively for the actual exposure time. We conclude that one count per jet area is likely to be due to contamination. This is considerably less than the Poisson uncertainty in our count numbers. These X-ray jet sources are highly significant (see discussion in Güdel et al. 2005, for the first exposure only). A linear feature pointing from the stellar PSF to the south, however, appears to be due to a coincidental arrangement of only four counts.

To characterize the jet morphology further, we smoothed the image using CIAO task aconvolve, treating the jets and the stellar PSF separately. In Fig. $1 \mathrm{~b}$ we used a Gaussian sigma of 0.75 pixels for the area outside the star, and 0.5 pixels for the stellar image. The smoothed image clearly shows the extended morphology of the two jet sources, reminiscent of the optical image at least for the SW jet (Dougados et al. 2000).

Figure 2 shows simulations of two different jet source shapes, based on the MARX software. In the left figure, the SW and NE jets are composed of a Gaussian with sigma $=1^{\prime \prime}$ and 0.8 , respectively, and the SW jet additionally contains a $1 \mathrm{D}$ linear source of $2^{\prime \prime}$ length, positioned radially outside the stellar $\mathrm{PSF}$ at a position angle of $225 \mathrm{deg}$. The linear feature contains $\approx 1.4$ times as many counts as the Gaussian source. The right figure shows a simulation in which both jet sources are point sources 5 .

\footnotetext{
${ }^{4} \mathrm{http}$ ://space.mit.edu/CXC/MARX: version 4.2 .0 was used.

5 The half-energy radius of the point response function of an ACIS-S on-axis source is about 0.41 or $\approx 1$ pixel, and the $80 \%$ encircledpower radius is $\approx 0 ! 7$ or nearly 2 pixels (see Chandra Proposers' Observatory Guide v.9). We also simulated the PSF in the Chandra Ray Tracer (ChART; Carter et al. 2002) software (http://cxc. harvard.edu/soft/ChaRT/cgi-bin/www-saosac.cgi) for the position of DG Tau and the boresight parameters of ObsID 4487 and 7246. The PSF was found to be compact, its core being very slightly elongated along a SE-NW axis, as can also be seen in Figs. 1 and 2. There is no extension in the directions of the jets (SW-NE).
}

The total number of counts in each simulated jet feature is similar to the numbers in the real $90 \mathrm{ks}$ observation. The observation resembles the simulation of extended sources; it is not compatible with one point source per jet. A more quantitative comparison will need longer exposures of these features.

We next generated "hardness" images, using two complementary procedures. In the first case, each individual count was attributed a color as a function of its energy. Energies in the $0.55-0.7 \mathrm{keV}$ range are represented in red, energies in the $1.5-1.75 \mathrm{keV}$ range in blue. In the $0.7-1.5 \mathrm{keV}$ range, colors change continuously from red to yellow to blue. The resulting image was smoothed, using a Gaussian with sigma $=0.85$ pixels - see Fig. 1c.

For an alternative representation, we extracted three pixel images smoothed as before (Gaussian smoothing with sigma $=0.75$ pixels), a "red", "green", and "blue" image for the $0.55-0.85 \mathrm{keV}, 0.85-1.2 \mathrm{keV}$, and $1.2-1.75 \mathrm{keV}$ ranges, respectively. The combined false-color image is shown in Fig. 1d.

Both hardness images show that the counter jet is harder, with photon energies mostly above $1 \mathrm{keV}$, while the forward jet shows a mixture of softer and harder counts.

\subsection{Spectral analysis of the jet sources}

Only simplistic models can be fitted to the spectra of the jets, given the small number of counts in the two jet sources. We experimented with isothermal plasma components (using the vapec model in XSPEC, with the abundances mentioned above) subject to photoelectric absorption (using the wabs model in XSPEC). Some plausible assumptions were made to keep the number of fit parameters low: Because the jet morphologies and the lengths of the two jets are similar, we adopted equal emission measures and (for similar heating mechanisms, e.g., shocks with similar velocity) equal temperatures. The absorption column densities, $N_{\mathrm{H}}$, remained different for each source because different gas components (e.g., the star's extended circumstellar disk) may absorb the jets differently, as also suggested by the hardness images discussed above. The initial fit parameters were therefore the emission measure (per jet), a single electron temperature (for both jets), and two $N_{\mathrm{H}}$ values. We found, however, that the absolute values of $N_{\mathrm{H}}$ are poorly constrained due to numerical cross-talk with the temperature (lower $N_{\mathrm{H}}$ can be compensated by higher temperatures), while the difference $\Delta N_{\mathrm{H}}=N_{\mathrm{H} \text {,counter }}-N_{\mathrm{H} \text {,forward }}$ between the absorption of the the counter jet and the forward jet is sufficiently well confined. This is illustrated in Fig. 3 where the confidence regions are shown on the $N_{\mathrm{H} \text {,counter }}$ vs. $N_{\mathrm{H} \text {,forward }}$ plane. The region of optimum $N_{\mathrm{H}}$ values shows a strong and nearly linear correlation between the two values, in such a way that their difference is well constrained. We have therefore chosen to fit $N_{\mathrm{H} \text {,forward }}$ and $\Delta N_{\mathrm{H}}$, while the value of $N_{\mathrm{H} \text {,counter }}$ can be derived from any pair of these two parameters.

The best-fit $\Delta N_{\mathrm{H}}$ is $2.7 \times 10^{21} \mathrm{~cm}^{-2}$, with a $1 \sigma$ range of $(1.6-4.2) \times 10^{21} \mathrm{~cm}^{-2}$ and a $90 \%$ range of $(0.9-5.2) \times 10^{21} \mathrm{~cm}^{-2}$. A differential absorption column between the two jets is thus very likely, and is in fact expected as discussed below. The plot shows two minima, the slightly deeper one located at $N_{\mathrm{H}, \text { forward }} \approx$ $0.02 \times 10^{22} \mathrm{~cm}^{-2}$ and $N_{\mathrm{H}, \text { counter }} \approx 0.29 \times 10^{22} \mathrm{~cm}^{-2}$, and a slightly shallower one at $N_{\mathrm{H} \text {,forward }} \approx 0.30 \times 10^{22} \mathrm{~cm}^{-2}$ and $N_{\mathrm{H} \text {,counter }} \approx 0.57 \times 10^{22} \mathrm{~cm}^{-2}$. The two solutions agree at the $1 \sigma$ level; however, the former solution requires a high bestfit temperature $(0.59 \mathrm{keV})$ which, together with an $N_{\mathrm{H}}$ value that is unusually low for Taurus pre-main sequence stars (Güdel et al. 2007a), suggests that the solution is unphysical. We adopt the second solution as the more reasonable "best-fit" in the 


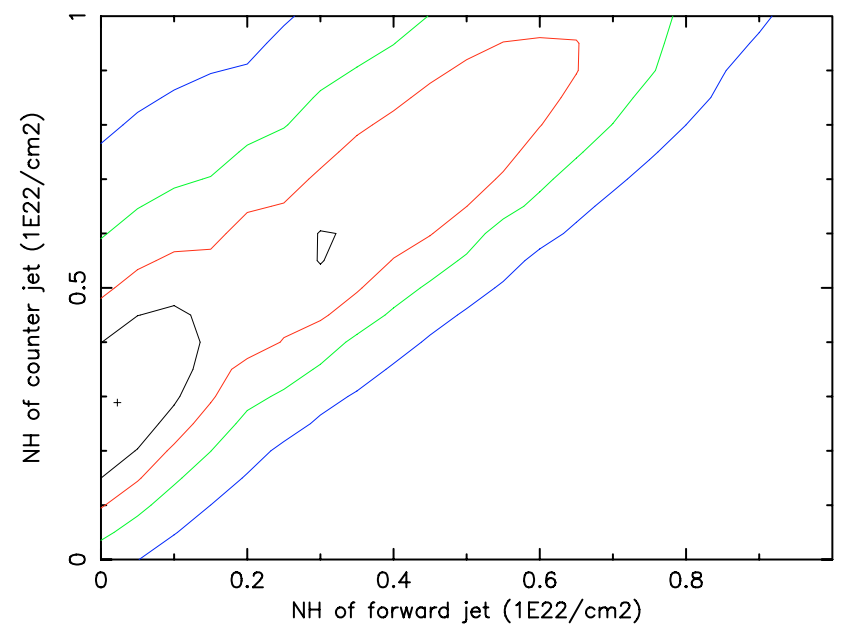

Fig. 3. Confidence regions for $N_{\mathrm{H}}$ of the counter jet versus $N_{\mathrm{H}}$ of the forward jet, using an isothermal plasma component per jet (with equal EMs and temperatures). The contours show the $1 \sigma$ (68\%, in red), $90 \%$, (in green), and $99 \%$ (in blue) confidence regions for two interesting parameters based on the $\mathrm{C}$ statistic. The black contour is plotted to outline the regions of lowest $C$ statistic (referring to $\Delta C=1$ ). The cross marks the location of the lowest $C$ statistic, although the minimum at $N_{\mathrm{H} \text {,forward }}=0.3$ and $N_{\mathrm{H} \text {,counter }}=0.57$ is more likely to represent a physical solution.

following although we repeat that only $\Delta N_{\mathrm{H}}$ can be sufficiently well constrained.

Despite the large errors of the fit parameters, three features are noteworthy. First, the absorption column density toward the forward (SW) jet is small $\left(\approx 3 \times 10^{21} \mathrm{~cm}^{-2}\right)$, agreeing with $N_{\mathrm{H}}$ of the soft but not the hard stellar component (see below). Second, the absorption toward the counter jet is higher, compatible with the increased hardness discussed in Sect. 4.1. And third, the electron temperature of the jet sources is low $(\approx 3.4 \mathrm{MK})$ compared with coronal temperatures of T Tauri stars (e.g., Telleschi et al. 2007b).

We do not give errors for $L_{X}$ as these depend very sensitively but non-trivially on the rather uncertain absorption column densities (see Güdel et al. 2007a, for estimates of lower limits to the uncertainties of $L_{X}$ of many X-ray sources in the Taurus star-forming region). Based on the best-fit values, the total X-ray output from the jets outside the stellar PSF (radius of $1^{\prime \prime}$. 4-1'. 7) would amount to a few times $10^{28} \mathrm{erg} \mathrm{s}^{-1}$ or $10-20 \%$ of the stellar soft component (see below).

\section{The DG Tau X-ray source}

The two-component spectral phenomenology of TAX sources (Güdel et al. 2007b) is present in each of the new Chandra observations. The spectral-fit results are reported in Table 3, together with the results from the XMM-Newton observation taken from Güdel et al. (2007b). The rather short exposures obtained in 2005-2006 led to considerable uncertainties in the derived parameters. This is particularly evident in the absorption column density, $N_{\mathrm{H}, 1}$, the electron temperature, $k T_{1}$, and consequently the X-ray luminosity, $L_{\mathrm{X}, 1}$, of the softer spectral component for the shortest observation, ObsID 7247, comprising only 142 cts. On the other hand, the count rates of the soft component agree within the error bars for all Chandra observations (Table 3), and no variability was recorded in the soft component during the individual exposures. Because the $90 \%$ error bars of $N_{\mathrm{H}, 1}$ and $k T_{1}$ also strongly overlap for the four Chandra exposures,

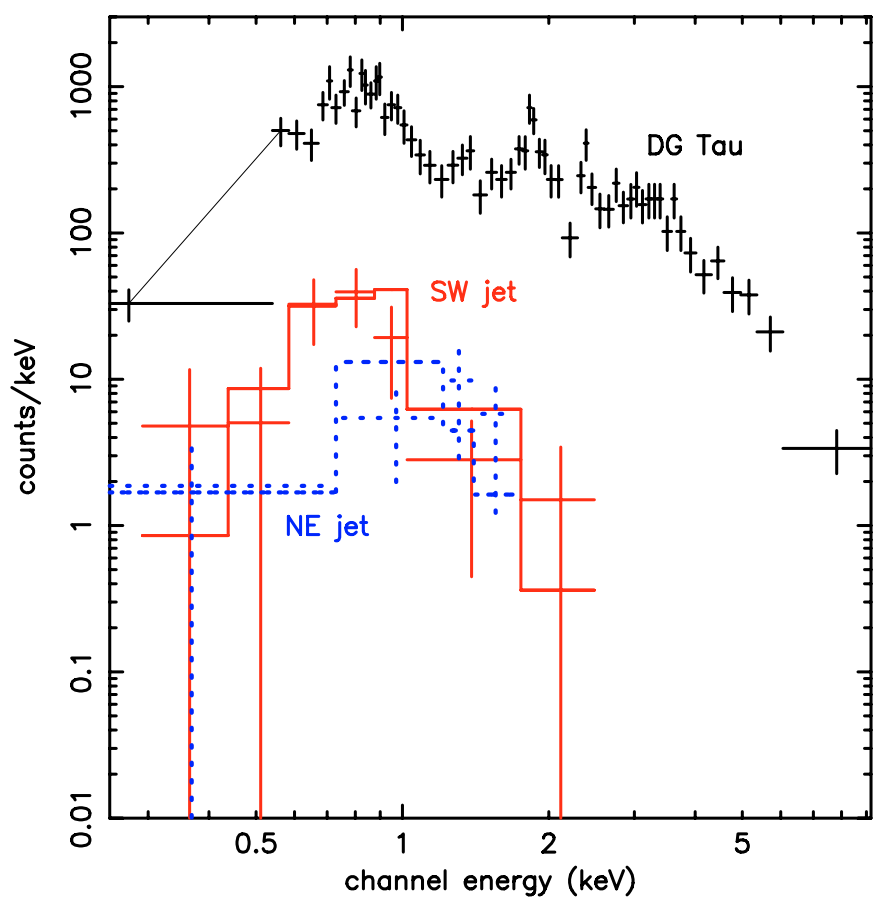

Fig. 4. Co-added ACIS-S spectra of the DG Tau point source (upper spectrum, black; the lowest-energy bins are connected for clarity) and the two jets (lower spectra; spectrum of less absorbed forward jet is shown solid, in red, while spectrum of the more strongly absorbed counter jet is shown dotted, in blue). For the jet sources, the solid histogram shows the joint spectral fit. The spectrum of DG Tau has been binned such that at least 15 counts are contained in each bin; the jet spectra have been binned arbitrarily to produce non-zero bins from $\approx 0.3 \mathrm{keV}$ to $\approx 2 \mathrm{keV}$.

we performed a joint fit of these spectra in the energy range of $0.2-1.1 \mathrm{keV}$, reported in the penultimate column in the table. The resulting parameters of the soft component compare very favorably with the XMM-Newton EPIC PN results (last column).

Because the hard component is variable (Table 3; slow, non-periodic modulations by a factor of $\approx 2$ were present in ObsID 7246 although larger flares were absent), agreement between the fit parameters is not expected, but we notice that the hydrogen absorption column density, $N_{\mathrm{H}, 2}$, agrees in all observations within the $90 \%$ error ranges. It is remarkable that $N_{\mathrm{H}, 2}$ is approximately 20 times higher than $N_{\mathrm{H}, 1}$.

For illustration purposes, we show the combined Chandra spectrum in Fig. 4 but again emphasize that spectral fits were performed for the individual, unbinned spectra (illustrations of spectral fits to binned data are shown in Güdel et al. 2005 for Chandra and in Güdel et al. 2007b for XMM-Newton).

\section{Discussion}

Our Chandra observations add unprecedented information to the X-ray emission model of DG Tau. In particular, we report the detection of an extended, bipolar X-ray jet down to the stellar PSF and out to a distance of about $5^{\prime \prime}$. This is the first doublesided X-ray jet reported from a pre-main sequence star.

The ACIS-S images reveal the presence of jets in the SW and NE directions, coincident with the direction of the optical jets. The NE jet appears to be slightly harder and also shows fewer photons in a similar extraction region. Further, the spectra of the DG Tau point source reveal a TAX spectral morphology. The DG Tau system thus hosts at least four distinct X-ray sources of 


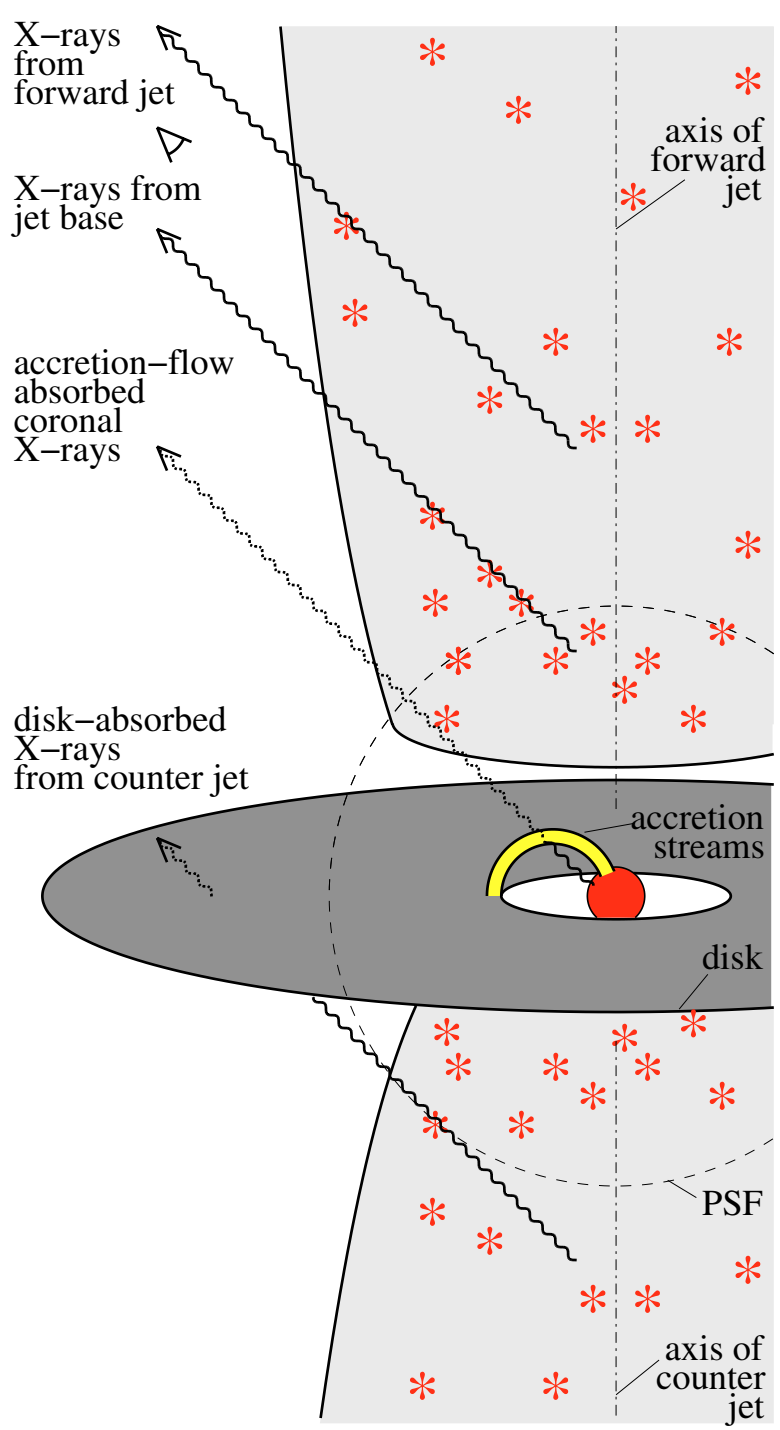

Fig. 5. Sketch of the proposed model of the DG Tau environment. Features are not drawn to scale. The observer is located toward the upper left. Four X-ray source regions are shown in red (the stellar coronal source, and asterisks symbolically marking emission regions in the jet) and their absorbing media are schematically shown. X-rays seen by the observer are sketched by the wavy lines, described on the left, from top to bottom: X-rays from the weakly absorbed, spatially resolved X-ray source of the forward jet; from the spatially unresolved but spectrally identified soft X-ray source closer to the base of the jet; from the hard, stellar coronal emission absorbed by the infalling accretion streams; and from the spatially resolved X-ray emission from the counter jet, slightly absorbed by the intervening disk gas. The dashed circle denotes the observing PSF of the star; features inside the PSF are unresolved from the stellar X-ray source.

different origin and subject to different gas absorption columns, as illustrated in the sketch shown in Fig. 5. These components are:

1. a weakly absorbed, diffuse, soft component along the forward-jet axis;

2. a more strongly absorbed, diffuse, soft component along the counter-jet axis;
3. a weakly absorbed, compact, non-variable, soft component (within the stellar PSF);

4. a strongly absorbed, compact, flaring, hard component (within the stellar PSF).

We now discuss the various features seen in the X-ray spectra and images, and propose a model consistent with all observed features.

\subsection{The X-ray jets: physical properties}

In this section, we will be interested as to how the jet cools, and why no X-ray emission is seen at distances beyond 5" from the star. There are further prominent bow shocks outside the regions discussed here, in particular a structure that was located at 10 .'7 from DG Tau in observations obtained in 1986 December (Eislöffel \& Mundt 1998) and still within $a \approx 12^{\prime \prime}$ a decade later (Stapelfeldt 1997). Using a proper motion of 0.'15-0.'3 per year (Eislöffel \& Mundt 1998; Dougados et al. 2000), this bow shock should now have expanded to about $15^{\prime \prime}$. Obviously, the DG Tau jet consists of a series of knots that are frequently ejected from the star (Eislöffel \& Mundt 1998). If the more distant knots were similar to those now seen in X-rays when at similar distances from the star, then the heated gas must have cooled, or the emission measure have decreased below our detection limit because we have found no significant $\mathrm{X}$-ray source at those distances. Given the time to travel from $\approx 5^{\prime \prime}$ to $\approx 11^{\prime \prime}$ (the region behind the outer bow shock, in the "X-ray free" lower right corner of Fig. 1), we adopt an X-ray decay time (with respect to the range of sensitivity of the X-ray detectors) of $\approx 20-40 \mathrm{yr}$.

To explore the potential cooling mechanism(s), we first discuss two extreme cases of cooling: cooling by expansion, i.e., adiabatic cooling; and radiative cooling without expansion. We will then discuss cooling if contributions from both cooling mechanisms contribute.

\subsubsection{Cooling by expansion}

The DG Tau jet significantly expands with increasing distance from the star. Transverse expansion has been measured; various features within a few arcsec of the star show an unusually large opening angle in the range of $\beta \approx 11-27$ degrees (based on full width at half maximum, after Dougados et al. 2000; see also Eislöffel \& Mundt 1998). In contrast, radial expansion (i.e., stretching of volumes in radial direction, along the linear flow) is unlikely to be important as this would require strong jet acceleration at distances of several arcseconds, or selective deceleration of regions closer to the star, for which no evidence has been reported.

There are two consequences of transverse expansion: a decrease of the EM and adiabatic cooling (in the absence of radiation), both leading to a decay of the X-ray emission in time.

The cross-section area of the jet at distance $r$ from the star is

$A(r)=\pi r^{2} \tan ^{2} \frac{\beta}{2}$.

or for small changes in $A(r)$,

$\frac{\mathrm{d} A}{A}=2 \frac{\mathrm{d} r}{r}$.

For the time span of 2 years, $\mathrm{d} r=0 .{ }^{\prime} 3-0 . ' 6$, and therefore $\mathrm{d} A / A \approx 0.12-0.24$ at a distance of $5^{\prime \prime}$. The electron density decreases by the same factor as the volume increases, and therefore 
the emission measure, $E M=n_{\mathrm{e}}^{2} V$, decreases by $12-24 \%$ at a distance of $\approx 5^{\prime \prime}$ within 2 years. Such a decrease is too small to be significantly measured in our 2004-2006 data.

However, for a distance of, say, $8^{\prime \prime}-11^{\prime \prime}$ (the lower-right area in Fig. 1), $A(r) / A\left(r_{0}\right)=\left(r / r_{0}\right)^{2} \approx 2.6-4.8$. The emission measure for a given mass element in the jet thus decreases by a factor of 2.6-4.8 from a distance of $5^{\prime \prime}$ to $8-11^{\prime \prime}$ from the star due to expansion alone. Because the gas is optically thin to X-ray emission, the X-ray surface brightness decreases by a factor of $2.6^{2}-4.8^{2}=6.8-23$, making its detection at this distance against the background level very difficult in our exposures.

At the same time, the plasma cools by expansion. In the limiting case, we neglect radiative losses, i.e., the gas cools adiabatically. Then,

$T V^{\gamma-1}=$ const.

with $\gamma=5 / 3$ for monatomic gas, and for small changes in $T$,

$$
\frac{\mathrm{d} T}{T}=(1-\gamma) \frac{\mathrm{d} V}{V}=-\frac{2}{3} \frac{\mathrm{d} A}{A}=-\frac{4}{3} \frac{\mathrm{d} r}{r}
$$

Using $\mathrm{d} A / A \approx 0.12-0.24$ for a two-year interval, we find $|\mathrm{d} T / T| \approx 0.08-0.16$. Again, this temperature decrease is too small to be measured during our observing interval.

For long intervals, however,

$$
\frac{T(r)}{T\left(r_{0}\right)}=\left(\frac{A(r)}{A\left(r_{0}\right)}\right)^{-2 / 3}=\left(\frac{r}{r_{0}}\right)^{-4 / 3}
$$

which, for $r=8^{\prime \prime}-11^{\prime \prime}$, the above projected jet velocities and $r_{0}=$ $5^{\prime \prime}$ yields $T(r) / T\left(r_{0}\right) \approx 0.35-0.53$, i.e. $T(r) \approx 1.2-1.8 \mathrm{MK}$.

A temperature decrease will also affect the count statistics in the detector, because the effective area decreases toward the softer energy range. We simulated the spectrum of the forward jet in XSPEC, adopting a much higher flux for better statistics and further changing its temperature. We then measured the simulated count rate in the $0.6-1.7 \mathrm{keV}$ range. Lowering $k T$ to $0.249-0.272 \mathrm{keV}(T=2.9-3.2 \mathrm{MK})$, which corresponds to $\mathrm{d} T / T \mid \approx 0.08-0.16$, reduces the count rate by $11-26 \%$. Together with the count rate drop from the decrease of the EM (see above), the total drop of the detected count rate would thus be $\approx 23-50 \%$. It is possible that this decrease affected our measured count rates, but we cannot prove such cooling effects as the count-rate decrease was not statistically significant during the two years covered by our observations. Furthermore, as some of the material in the detected jet structure may cool, new hot material is likely to be replenished from closer to the star, as we have found the $\mathrm{X}$-ray structure to be extended toward the stellar PSF.

On the other hand, considering a longer time span of 20 years, corresponding to an expansion from $5^{\prime \prime}$ to $8^{\prime \prime}-11^{\prime \prime}$, therefore lowering the plasma temperature to $1.8 \mathrm{MK}$, the count rate decreases by $83 \%$. This, together with the strong decrease of the EM, reinforces our finding that a cooling, expanding X-ray jet starting under presently observed conditions at 5 " distance will be difficult to detect at distances of $8-11^{\prime \prime}$, i.e., the region just behind a bow shock described by Eislöffel \& Mundt (1998).

In summary, adiabatic expansion alone should lead to the disappearance of the X-ray jet at distances significantly beyond $5^{\prime \prime}$ in Chandra exposures such as ours. Our exposures are not sufficient, on the other hand, to verify a cooling effect during the two-year time span.
Table 2. Results from spectral fit to jet sources. Errors give $1 \sigma$ confidence ranges, and EM and $L_{\mathrm{X}}$ values are for one jet each.

\begin{tabular}{lcc}
\hline \hline & SW Jet: & NE Jet: \\
\hline$N_{\mathrm{H}}^{a, b}$ & $3.0_{-3.0}^{+3.0} \times 10^{21} \mathrm{~cm}^{-2} \quad 5.7 \times 10^{21} \mathrm{~cm}^{-2}$ \\
$\Delta N_{\mathrm{H}}$ & $2.7_{-1.1}^{+1.5} \times 10^{21} \mathrm{~cm}^{-2}$ \\
$T^{b}$ & $3.4_{-1.1}^{+5.2} \times 10^{6} \mathrm{~K}$ \\
$\mathrm{EM}^{b}$ & $1.0_{-0.8}^{+6.3} \times 10^{51} \mathrm{~cm}^{-3}$ \\
$L_{\mathrm{X}}[0.1-10 \mathrm{keV}]$ & $1.2 \times 10^{28} \mathrm{erg} \mathrm{s}^{-1}$ \\
\hline
\end{tabular}

a The two $N_{\mathrm{H}}$ values are coupled through the fit parameter $\Delta N_{\mathrm{H}}$. Therefore, no independent errors are given for the NE jet; ${ }^{b} N_{\mathrm{H}, \text { forward }}$ and $\mathrm{EM}$ are, within $1 \sigma$, poorly constrained to low values and $T$ is poorly constrained to high values because there is a second (probably unphysical) minimum in the statistic, with $N_{\mathrm{H} \text {,forward }} \approx 0, T=6.8 \mathrm{MK}$, and very small EM. See text for details.

\subsubsection{Cooling by radiation}

Radiative cooling may be significant as well. We cannot estimate the radiative cooling time because the plasma density is unknown, or in other words, it is not clear whether the observed $\mathrm{X}$-ray radiation extracts a significant fraction of the thermal energy of the hot plasma within the 2-20 yr of interest here. We will, on the other hand, study consequences for the X-ray jet if radiative cooling is the dominant loss mechanism. The volume of the X-ray detected source of the forward jet is approximately (see Sect. 4.1)

$$
V=\frac{3^{\prime \prime} *(0.5)^{2} \pi}{\sin (38 \mathrm{deg})} *\left(2.1 \times 10^{15}\right)^{3} \mathrm{~cm}^{3},
$$

where $38 \mathrm{deg}$ is the jet inclination (Eislöffel \& Mundt 1998), we have adopted a length outside the PSF and a cross-sectional radius of the X-ray jet of $3^{\prime \prime}$ and 0.5 , respectively (Sect. 4.1), and the final constant gives the conversion from arcseconds to $\mathrm{cm}$ at the distance of Taurus. We thus find $V=3.6 \times 10^{46} \mathrm{~cm}^{-3}$ but note that the X-ray gas may occupy only a fraction of this volume, defined by the volume filling factor $f$.

With the best-fit EM from Table 2, the density of the X-ray emitting jet gas is

$n_{\mathrm{e}}=\left(\frac{\mathrm{EM}}{f V}\right)^{1 / 2} \approx \frac{170}{f^{1 / 2}}\left[\mathrm{~cm}^{-3}\right]$.

The thermal energy decay time then is, assuming that energy is lost by radiative cooling (i.e., decrease of $T$ ) only,

$$
\tau=\frac{3 k T}{n_{\mathrm{e}} \Lambda(T)} \approx 1.4 \times 10^{5} T f^{1 / 2}[\mathrm{~s}] \approx 15400 f^{1 / 2}[\mathrm{yr}]
$$

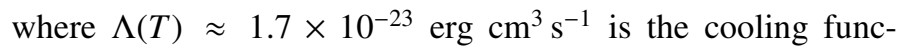
tion (evaluated at 3.4 MK for our model, derived from apec for the $0.001-100 \mathrm{keV}$ interval), and in the last equation we have adopted the X-ray measured jet temperature of 3.4 MK.

Here, the energy decay reflects exclusively in a decrease of the temperature, $T$, while the emission measure remains constant. As before, we model the count rate reduction for decreasing temperatures for the ACIS-S detector. Given that we detected 18 cts in the forward jet, we now seek the temperature for which the same emission measure results in a detection limit of only 5-6 detected counts, i.e., a count rate reduced to 25-30\%. For fewer counts, the source will be difficult to detect, depending also on the spatial distribution of the counts (we recall that approximately one count will be due to contamination from background radiation and the stellar PSF). Using the detector response, we found that a count rate reduction to $25-30 \%$ 
Table 3. Results from spectral fits for the DG Tau point source. Errors give $90 \%$ confidence limits.

\begin{tabular}{|c|c|c|c|c|c|c|}
\hline \multirow[b]{2}{*}{ ObsID } & \multicolumn{5}{|c|}{ Chandra } & \multirow{2}{*}{$\frac{\text { XMM-Newton }}{0203540201^{a}}$} \\
\hline & 4487 & 6409 & 7247 & 7246 & all Chandra & \\
\hline Epoch & Jan. 2004 & Dec. 2005 & Dec. 2005 & Apr. 2006 & 2004-2006 & Aug. 2004 \\
\hline counts $[0.2-7 \mathrm{keV}]$ & 388 & 204 & 142 & 349 & - & 634 \\
\hline count rate $\left[\mathrm{ct} \mathrm{s}^{-1}, 0.2-7 \mathrm{keV}\right]^{b}$ & $0.0131(7)$ & $0.0126(9)$ & $0.0089(7)$ & $0.0125(7)$ & - & $0.0206(10)$ \\
\hline $\begin{array}{l}\text { Soft component: } \\
\text { count rate }\left[\mathrm{ct} \mathrm{s}^{-1}, 0.2-1.1 \mathrm{keV}\right]^{b}\end{array}$ & $0.0046(4)$ & $0.0041(5)$ & $0.0041(5)$ & $0.0046(4)$ & - & $0.0083(6)$ \\
\hline$N_{\mathrm{H}, 1}\left[10^{21} \mathrm{~cm}^{-2}\right]$ & $1.4_{-1.0}^{+1.8}$ & $0.95_{-0.93}^{+2.3}$ & $4.5_{-1.9}^{+4.1}$ & $2.0_{-1.7}^{+2.6}$ & $1.3_{-0.6}^{+1.1}$ & $1.1_{-0.8}^{+1.4}$ \\
\hline $\begin{array}{l}T_{1}[\mathrm{MK}] \\
L_{\mathrm{X}, 1}\left[\mathrm{erg} \mathrm{s}^{-1}, 0.1-10 \mathrm{keV}\right]\end{array}$ & $\begin{array}{l}4.6_{-1.3}^{+2.3} \\
1.1 \times 10^{29}\end{array}$ & $\begin{array}{l}6.0_{-2.7}^{+1.7} \\
8.2 \times 10^{28}\end{array}$ & $\begin{array}{l}3.2_{-1.5}^{+1.5} \\
4.5 \times 10^{29}\end{array}$ & $\begin{array}{l}3.6_{-1.0}^{+2.3} \\
1.7 \times 10^{29}\end{array}$ & $\begin{array}{l}4.5_{-0.9}^{+0.7} \\
1.0 \times 10^{29}\end{array}$ & $\begin{array}{l}3.7_{-0.5}^{+0.9} \\
0.96 \times 10^{29}\end{array}$ \\
\hline $\begin{array}{l}\text { Hard component: } \\
\text { count rate }\left[\mathrm{ct} \mathrm{s}^{-1}, 1.7-7 \mathrm{keV}\right]^{b}\end{array}$ & $0.0063(5)$ & $0.0068(6)$ & $0.0031(4)$ & $0.0066(5)$ & - & $0.0099(7)$ \\
\hline$N_{\mathrm{H}, 2}\left[10^{21} \mathrm{~cm}^{-2}\right]$ & $21_{-6}^{+8}$ & $32_{-11}^{+16}$ & $15_{-10}^{+14}$ & $33_{-12}^{+11}$ & - & $18_{-6}^{+6}$ \\
\hline $\begin{array}{l}T_{2}[\mathrm{MK}] \\
L_{\mathrm{X}, 2}\left[\mathrm{erg} \mathrm{s}^{-1}, 0.1-10 \mathrm{keV}\right]\end{array}$ & $\begin{array}{l}31_{-9}^{+21} \\
6.9 \times 10^{29}\end{array}$ & $\begin{array}{l}27_{-10}^{+25} \\
1.0 \times 10^{30}\end{array}$ & $\begin{array}{l}34_{-16}^{+77} \\
2.8 \times 10^{29}\end{array}$ & $\begin{array}{l}23_{-6}^{+23} \\
1.1 \times 10^{30}\end{array}$ & - & $\begin{array}{l}69_{-25}^{+119} \\
9.6 \times 10^{29}\end{array}$ \\
\hline
\end{tabular}

${ }^{a}$ Average properties for the hard component during XMM-Newton EPIC PN observations (including flares); counts for $24932 \mathrm{~s}$ of low-background data; ${ }^{b}$ parentheses give errors in last digit.

corresponds to a temperature reduction to $50-61 \%$ of the initial value, i.e., a time interval of $0.49-0.56$ e-folding decay times of the energy (Eq. (8)).

If the jet should become undetectable in an observation like ours after 20-40 yr, i.e., for an energy e-folding decay time of $10-22 \mathrm{yr}$, we require $f=4 \times 10^{-7}-2 \times 10^{-6}$, or densities of $(1.2-2.6) \times 10^{5} \mathrm{~cm}^{-3}$.

\subsubsection{Cooling by expansion and radiation}

If radiative losses are significant during the time span of interest here, then the adiabatic approximation in Sect. 6.1.1 breaks down. On the other hand, if we accept that the hot plasma is subject to expansion as observed for the cooler optical jet, then the treatment of radiative losses in Sect. 6.1.2 is also not sufficient. Both cooling terms must be combined.

We consider the first law of thermodynamics,

$$
\mathrm{d} U+\delta W=\delta Q \text {. }
$$

Here, $\mathrm{d} U$ is the change of the internal energy, $U=2 \alpha N_{\mathrm{e}} k T$, where $N_{\mathrm{e}}$ is the total number of electrons and $\alpha=1 /(\gamma-1)=3 / 2$ for monatomic gas (for simplicity, we adopt a hydrogen plasma with a total number of particles $N=N_{\mathrm{p}}+N_{\mathrm{e}}=2 N_{\mathrm{e}}$; the difference to a realistic plasma is not significant, the electron density in the latter being only about $10 \%$ larger than the ion density). Further, $\delta W=p \mathrm{~d} V$ is the pressure work done by the gas, with a pressure of $p=2 n_{\mathrm{e}} k T$. If radiative losses are present, we set

$\delta Q=-n_{\mathrm{e}}(t)^{2} V(t) \Lambda(T) \mathrm{d} t$

where $\Lambda(T)$ is the temperature-dependent cooling function (in erg $\mathrm{cm}^{3} \mathrm{~s}^{-1}$ ) and $n_{\mathrm{e}}$ is the electron density. Substituting these expressions into Eq. (9), we find

$\alpha \frac{\mathrm{d} T}{T(t)}+\frac{\mathrm{d} V}{V(t)}=-\frac{n_{\mathrm{e}}(t) \Lambda(T)}{2 k T(t)} \mathrm{d} t$.

Note that in the absence of radiative losses, Eq. (11) is equivalent to Eqs. (3) and (4) for an adiabatic process. Radiation makes the problem explicitly time-dependent; the initial electron density, $n_{0}=n_{\mathrm{e}}(t=0)$, is introduced as a free parameter.

We define the observed expansion of a given volume element by the radial distance along the jet axis, $r(t)=r_{0}+v t$. Then,

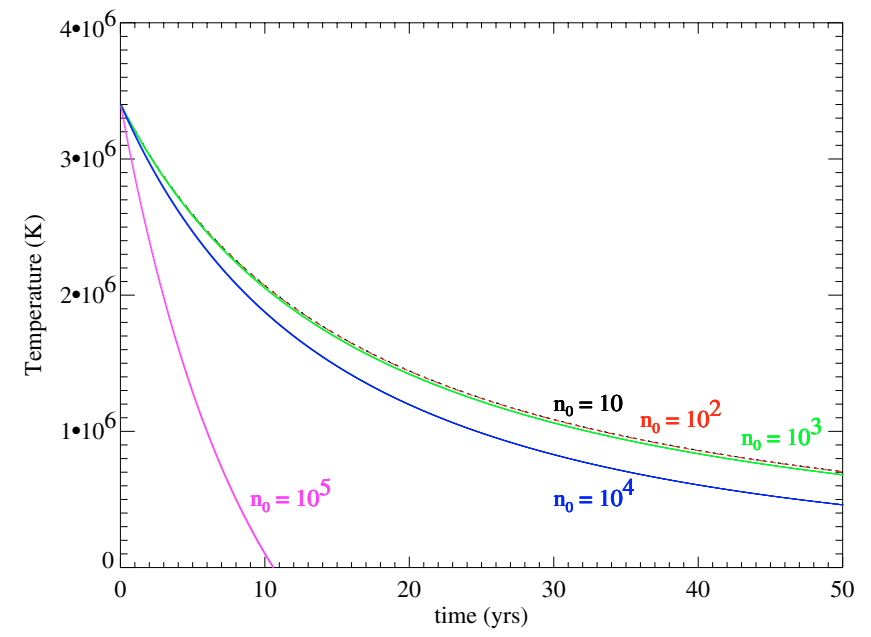

Fig. 6. Cooling behavior of the jet plasma, assuming transverse expansion as observed in the optical jet, and radiative losses for various initial electron densities, $n_{0}=n_{\mathrm{e}}(t=0)$ at a stellar distance of $r_{0}=5^{\prime \prime}$. The curves are color-coded for initial densities given in the plot. The two curves for $n_{0}=10 \mathrm{~cm}^{-3}$ and $n_{0}=100 \mathrm{~cm}^{-3}$ are nearly coincident.

$V \propto r^{2}$ (in the absence of expansion along the jet axis), and $n_{\mathrm{e}} \propto V^{-1} \propto r^{-2}$. Substituting these expressions, we find

$\alpha \frac{\mathrm{d} T}{T(t)}=-\left[\frac{2 v}{r(t)}+\left(\frac{r_{0}}{r(t)}\right)^{2} \frac{n_{0} \Lambda(T)}{2 k T(t)}\right] \mathrm{d} t$.

We have determined the run of $T$ numerically, starting with $T_{0}=3.4 \mathrm{MK}$ and with various $n_{0}$ values, taken to be the initial electron density at $r_{0}=5^{\prime \prime}$ (not yet considering a strict lower limit to $n_{0}-$ see below). The cooling function was approximated by $\Lambda(T)=4.83 \times 10^{-23}-9.75 \times 10^{-24} T \mathrm{erg} \mathrm{cm}^{3} \mathrm{~s}^{-1}$ ( $T$ in MK) for the range of $T=1-3.5 \mathrm{MK}$, as found from an integration of the spectral energy distribution for an isothermal plasma in XSPEC, using the vapec code with our adopted element abundances (Sect. 3). For $T<1 \mathrm{MK}$, we kept $\Lambda$ constant at $4 \times 10^{-23} \mathrm{erg} \mathrm{cm}^{3} \mathrm{~s}^{-1}$. We used an average projected jet velocity of $0.225^{\prime \prime} \mathrm{yr}^{-1}$. The results are shown in Fig. 6. We see that - in agreement with the estimates for pure radiative losses the cooling process is dominated by expansion up to densities of 
several times $10^{3} \mathrm{~cm}^{-3}$, i.e., cooling is adiabatic. Only for densities $\gtrsim 10^{4} \mathrm{~cm}^{-3}$ does radiation matter on time scales of tens of years.

\subsubsection{Implications from jet cooling}

Because the electron density in the jet X-ray source is unknown, the importance of radiative cooling cannot be directly assessed. We will, however, discuss the limiting case of low densities. Equation (7) gives the lowest density for the maximum volume filling factor, $f=1$, namely $n_{\mathrm{e}}=170 \mathrm{~cm}^{-3}$. This implies an upper limit for the thermal energy of the hot gas of $3 n_{\mathrm{e}} k T V \approx$ $8.6 \times 10^{39}$ erg in the forward jet alone or $\approx 1.7 \times 10^{40} \mathrm{erg}$ in both jets together.

The mass outflow rate in the DG Tau jets is $6 \times 10^{-8} M_{\odot} \mathrm{yr}^{-1}$ (twice the value given by Lavalley-Fouquet et al. 2000 for the forward jet) with a characteristic flow velocity of $300 \mathrm{~km} \mathrm{~s}^{-1}$ (e.g., Eislöffel \& Mundt 1998; Dougados et al. 2000). The kinetic energy rate is therefore $\dot{M}_{\text {jet }} v_{\text {jet }}^{2} / 2 \approx 1.7 \times 10^{33} \mathrm{erg} \mathrm{s}^{-1}$. The total jet X-ray luminosity is, according to Table 2, $2.4 \times$ $10^{28} \mathrm{erg} \mathrm{s}^{-1}$, i.e., a fraction of $1.4 \times 10^{-5}$ of the kinetic energy dissipates in X-rays after transformation to thermal energy of the hot plasma.

The jet out to a distance of $5^{\prime \prime}$ forms within $\approx 20 \mathrm{yr}$ based on the measured proper motion. The mass outflow rate then indicates that $\approx 1.2 \times 10^{-6} M_{\odot}$ are contained in the jets, with a total kinetic energy of $1.1 \times 10^{42} \mathrm{erg}$. Therefore, a maximum of $1.6 \%$ of the kinetic energy is transformed into thermal energy in the hot plasma.

We can now investigate whether the pressure in the hot gas contributes to the expansion of the jet. The bulk gas observed in optical lines has a density of $10^{3} \mathrm{~cm}^{-3}-10^{4} \mathrm{~cm}^{-3}$ at distances of a few arcsec from the star, with an ionization fraction approaching unity (Lavalley-Fouquet et al. 2000). The temperature is of order $10^{4} \mathrm{~K}$ (Hamann 1994). The gas pressure is therefore of order $P_{\text {bulk }} \approx\left(3 \times 10^{-9}-3 \times 10^{-8}\right) \mathrm{dyn}^{-2}$. For the X-ray emitting gas, we estimate, from the above values, a minimum pressure for $f=1$, namely $P_{\text {hot }} \approx\left(1.6 \times 10^{-7}\right)$ dyn $\mathrm{cm}^{-2}$. A filling factor of unity is impossible because only a small fraction of the energy heats the hot plasma while cooler gas predominates. It thus appears that the hot gas pressure is grossly out of equilibrium with its environment, contributing to the transverse jet expansion discussed in this section.

We emphasize that this argumentation holds regardless of the exact filling factor or of the relevance of radiative cooling. It is a minimum-density estimate related to the energy decay in Eq. (8).

\subsection{Heating the $X$-ray jets}

How are the jets heated to the observed temperatures? Most of the conventionally detected emission from jets of pre-main sequence stars comes from low-ionization transitions such as [O I], $[\mathrm{N}$ II] , or $[\mathrm{S}$ II $]$, indicating temperatures of no more than a few thousand K. Hotter gas has been identified, e.g., in emission from He I, [O III], C III, and O IV, giving evidence for hot winds (Cohen \& Fuller 1985; Takami et al. 2002; Dupree et al. 2005) although some of these claims have been questioned, and maximum wind temperatures of only $2 \times 10^{4} \mathrm{~K}$ have been derived (Johns-Krull \& Herczeg 2007; Kwan et al. 2007). Measured shock velocities in the jets of DG Tau are of order $50-100 \mathrm{~km} \mathrm{~s}^{-1}$ (Lavalley-Fouquet et al. 2000), sufficient to heat gas to a few $10^{5} \mathrm{~K}$ but not to the observed 3-5 MK.
A possibility is that a fraction of the jet gas collides directly with the interstellar medium, in which case the shock velocity would be several $100 \mathrm{~km} \mathrm{~s}^{-1}$ and therefore in principle sufficient to heat a fraction of the gas to X-ray emitting temperatures. Alternatively, magnetic heating may be in operation. Magnetic fields are commonly invoked to explain jet acceleration near the star and the disk, and also to collimate jets during their propagation (Blandford \& Payne 1982; Uchida \& Shibata 1985; Königl \& Pudritz 2000; Shu et al. 2000).

Ambipolar diffusion heating results from the separation of charged particles from neutrals across magnetic fields. The process has been described in detail by Safier (1992), Garcia et al. (2001a), and Garcia et al. (2001b). It is most efficient as long as the gas is weakly ionized and therefore not relevant for our hot plasma. Self-consistent jet models converge to jet temperatures of $\approx 10^{4} \mathrm{~K}$ due to ambipolar diffusion heating.

Ohmic dissipation of currents operates in highly ionized gas. Initially jet-aligned magnetic fields are wound up due to rotation, producing helical fields which drive currents. Depending on $\nabla \times \boldsymbol{B}$, sufficient dissipation may be achieved to heat the gas to high temperatures. In order for Ohmic dissipation to become effective, the gas should be pre-heated, which could be achieved by shocks. Direct magnetic-field measurements in the jets of DG Tau would be important to quantitatively assess the heating mechanism, e.g., based on radio synchrotron emission from accelerated electrons (Curiel et al. 1993; Ray et al. 1997).

\subsection{The gas-to-dust ratio in the outer disk of DG Tau}

The hardness difference between the forward and the counter jet is easily explained by the presence of a gas disk that absorbs the softest photons from the counter jet (see Fig. 5). This observation therefore in principle offers the opportunity to measure the gas-to-dust ratio in a circumstellar disk (at distances of a few hundred $\mathrm{AU}$ ) from differential absorption and extinction measurements. The spectral-fit results summarized in Table 2 show a difference between the gas column densities of the two jets of $\Delta N_{\mathrm{H}} \approx 2.7 \times 10^{21} \mathrm{~cm}^{-2}$, albeit with large errors. For standard assumptions pertaining to the interstellar medium (e.g., dust grain size distribution, atomic gas absorption with "solar" elemental composition), $N_{\mathrm{H}} \approx 2 \times 10^{21} A_{\mathrm{V}}\left[\mathrm{cm}^{-2}\right]$ (Vuong et al. 2003). Therefore, $\Delta N_{\mathrm{H}}$ corresponds to a visual extinction difference of $\Delta A_{\mathrm{V}} \approx 1.4 \mathrm{mag}$ (with a $1 \sigma$ range of $\approx 0.8-2.1 \mathrm{mag}$ ). The difference between the visual extinctions of the two jets is also not precisely known; Lavalley et al. (1997) estimate a difference of about 3 mag at a distance of $1^{\prime \prime}$ from the star, which would suggest nearly standard gas-to-dust ratios when combined with $\Delta N_{\mathrm{H}}$, while Pyo et al. (2003) propose a difference of as much as $14.2 \mathrm{mag}$ at the same position. Better determinations of differential gas absorption and visual extinction are needed; we also note that most of the jet-related X-ray counts were collected from larger stellar distances $\left(2^{\prime \prime}-5^{\prime \prime}\right)$ than the distances to which the $\Delta A_{\mathrm{V}}$ values refer.

\subsection{The hard stellar source}

Because the hard component occasionally flares, in one case being preceded by $U$ band emission as in solar and stellar flares (Güdel et al. 2007b), it is most straightforwardly interpreted as coronal or "magnetospheric". The high temperatures are also not consistent with shock heating given gas flow velocities of no more than a few $100 \mathrm{~km} \mathrm{~s}^{-1}$ (as observed in the jets, or inferred from free-fall onto the stellar surface). 
The hard component of the DG Tau point source is unusually strongly absorbed, with $N_{\mathrm{H}} \approx 2 \times 10^{22} \mathrm{~cm}^{-2}$. From the stellar extinction, $A_{\mathrm{V}}=1.5-3 \mathrm{mag}$ (Güdel et al. $2007 \mathrm{~b}$ and references therein), we expect $N_{\mathrm{H}} \approx(3-6) \times 10^{21} \mathrm{~cm}^{-2}$. We therefore find from Table 3 , that the dust content in the accretion streams is depleted by factors of 3-6.

The excess photoelectric absorption requires the presence of gas that is depleted of dust in order to suppress optical extinction. One possibility are relatively cool stellar or disk winds. However, as they expand to large stellar distances, they would also affect the jet components and the soft "stellar" component unless these were placed very far from the star. Also, it is not clear why such winds would be dust-depleted, or if they initially are, why they would not form dust.

Dust is destroyed at several stellar radii due to stellar irradiation; for DG Tau in particular, Güdel et al. (2007b) estimated the dust sublimation radius at $(7-10) R_{*}$, similar to the corotation radius (see below). The region inside the corotation radius is thought to be dominated by magnetic accretion. The excess absorption is thus most easily explained by the infalling, dust-depleted massive accretion streams. These observations therefore provide indirect evidence for dust-depleted accretion streams that absorb the X-ray emission from the underlying corona.

We now show that the amount of photoelectric absorption is plausible. Estimating the gas column density along the line of sight through the accretion streams to the stellar corona requires knowledge of the accretion geometry, which is not available. As a conservative limit, we assume spherically symmetric infall. The corotation radius, at which the mass begins to accelerate toward the star, can be derived from Kepler's law:

$a_{\text {corot }}=\left(\frac{G M_{*} P^{2}}{4 \pi^{2}}\right)^{1 / 3}=9.2 \times 10^{11} \mathrm{~cm}=5.6 R_{*}$

where $M_{*}=0.91 M_{\odot}$ is the stellar mass (Briceño et al. 2002), $R=2.46 R_{\odot}$ is the stellar radius (Güdel et al. 2007a), $P=6.3 \mathrm{~d}$ is the rotation period (Bouvier et al. 1993), and $G=6.673 \times$ $10^{-8}$ dyn $\mathrm{cm}^{2} \mathrm{~g}^{-2}$ is the constant of gravitation. For a semicircular funnel stream guided by magnetic fields, the highest elevation is reached midway between the corotation radius and the stellar surface, at $r=3.3 R_{*}$. There, the stream's velocity, $v_{\text {in }}=1.3 \times 10^{7} \mathrm{~cm} \mathrm{~s}^{-1}$ if free-fall acceleration is assumed (as an upper limit) starting from the corotation radius, is about $40 \%$ of the fall velocity at the stellar surface, and the ionization degree of the gas may still be moderate. We note that the jet-axis inclination and by inference the most likely stellar rotation-axis inclination is $\approx 38 \mathrm{deg}$ (Eislöffel \& Mundt 1998). For an orderof-magnitude estimate, we assume spherical infall at this radius, over a radial distance of $1 R_{*}$. The mass accretion rate in this case is, for spherical symmetry

$\dot{M} \approx 4 \pi r^{2} n_{\mathrm{H}} m_{\mathrm{p}} v_{\text {in }} \approx 8.9 \times 10^{7} n_{\mathrm{H}}$

where $n_{\mathrm{H}}$ is the hydrogen number density, and $m_{\mathrm{p}}$ is the proton mass; we assume a mean mass per particle of $m_{\mathrm{p}}$. The observed accretion rate is $\dot{M}=\left(10^{-7.34}-10^{-6.13}\right) M_{\odot} \mathrm{yr}^{-1}$ (White \& Ghez 2001; White \& Hillenbrand 2004). We thus find $n_{\mathrm{H}} \approx 3.3 \times$ $10^{10} \mathrm{~cm}^{-3}-5.3 \times 10^{11} \mathrm{~cm}^{-3}$. Integrated over one stellar radius, the absorption column density is $N_{\mathrm{H}} \approx 5.7 \times 10^{21}-9.1 \times 10^{22} \mathrm{~cm}^{-2}$, in agreement with the measured values of order $2 \times 10^{22} \mathrm{~cm}^{-2}$.

More confined or slower accretion streams will produce higher densities, while ionized gas will reduce photoelectric absorption. We emphasize that the high accretion rate is crucial to obtain high $N_{\mathrm{H}}$ in the above estimates. All TAX sources reported before (Güdel et al. 2007b) are indeed very strongly accreting $\mathrm{T}$ Tauri stars.

\subsection{The soft "stellar" source}

The soft emission commonly seen in X-ray spectra from other $\mathrm{T}$ Tauri stars, formed at a few MK as part of a wide distribution of plasma in the magnetic corona (e.g., Preibisch et al. 2005), is not detected here but is absorbed, leaving only a "hard" coronal component above $1.5 \mathrm{keV}$ in our spectra. In contrast, we see a very strong, separate soft component that is not related to the coronal spectrum as judged from its very different absorption and the absence of variability during flares seen in the coronal component at higher photon energies (Güdel et al. 2007b). The strong absorption of the coronal X-ray component makes an origin of the soft spectral emission from a location close to the stellar surface unlikely. In fact, our new observations suggest that the gas absorption of the soft X-ray component, $N_{\mathrm{H}}=1.3(0.7-2.4) \times 10^{21} \mathrm{~cm}^{-2}(90 \%$ error range; Table 3), is lower than the absorption suggested from the visual extinction to the star, $N_{\mathrm{H}}\left(A_{\mathrm{V}}\right) \approx(3-6) \times 10^{21} \mathrm{~cm}^{-2}$ (based on $A_{\mathrm{V}} \approx 1.5-3 \mathrm{mag}$, see White \& Ghez 2001; White \& Hillenbrand 2004; Muzerolle et al. 1998; and Hartigan et al. 1995). A likely origin of these X-rays is the base of the forward jet. Such an origin is suggested by $i$ ) the unusually soft emission not usually seen in T Tauri stars (Güdel et al. 2007a), ii) the low $N_{\mathrm{H}, 1}$, and iii) the explicit evidence for jets in the Chandra image. Further, $N_{\mathrm{H}, 1}$ and $k T_{1}$ agree with the corresponding values of the forward jet (Table 2). We therefore suggest that the jets continue to be X-ray sources to smaller stellar distances, producing an order of magnitude more soft X-rays within the Chandra point-spread function of $\approx 2^{\prime \prime}$ radius than outside this radius.

\section{Summary and conclusions}

We have unambiguously detected a bipolar X-ray jet associated with the strongly accreting classical T Tauri star DG Tau. This is the first bipolar X-ray jet reported from a pre-main sequence star. The jet is extended at Chandra's spatial resolution and can be followed down into the PSF of DG Tau itself. The jets are roughly symmetric as far as length and structure are concerned, reaching out to about $5^{\prime \prime}$ from the star.

The X-ray emitting gas of the jet is relatively cool, $T \approx$ 3.4 MK, which however still poses a problem for our understanding of the heating mechanism. Shock velocities in this jet are too small to heat gas to such temperatures. Ohmic heating by magnetic-field driven, dissipating currents may be an alternative.

We find various gas absorption columns toward the four X-ray components detected in the DG Tau system. The soft stellar component shows $N_{\mathrm{H}}$ smaller than the value derived from the stellar visual extinction assuming standard gas-to-dust ratios. This suggests that the soft spectral emission originates from a region "in front" of the star, i.e., we identify the soft component with X-ray emission similar to that from the forward jet but produced too close to the star to be resolved in the Chandra images.

In contrast, the counter jet suggests stronger absorption, which is expected because its X-rays traverse the extended outer gas disk. A determination of the gas-to-dust ratio is therefore in principle possible by measuring the differential absorption and extinction of the two jets. We have succeeded in determining the difference in $N_{\mathrm{H}}$ although not the absolute values of each column density. The gas column of the intervening extended disk structure is relatively small $\left(2.7 \times 10^{21} \mathrm{~cm}^{-2}\right)$, corresponding to 
1.5-2 mag of visual extinction for standard gas-to-dust ratios. An extinction difference of $3 \mathrm{mag}$ has been reported in a previous paper (Lavalley et al. 1997), i.e., the outer-disk composition is nearly compatible with interstellar gas-to-dust ratios. However, more accurate determination both of the absorption difference and the visual extinction difference is needed.

Finally, the hard component in the stellar spectrum is attributed to a (flaring) corona. The excess photoelectric absorption is ascribed to accretion gas streams that are dust depleted because the dust destruction radius is similar to the radius of the inner disk edge, thought to be close to the corotation radius.

Why is the detection of X-ray jets important? The combined power of the resolved jets and the unresolved soft spectral component is of order $10^{29} \mathrm{erg} \mathrm{s}^{-1}$ or similar to the X-ray output of a relatively $\mathrm{X}$-ray faint $\mathrm{T}$ Tauri star. This emission is distributed above the inner accretion disk. It is therefore an important contributor to X-ray heating and ionization of gaseous disk surfaces (Glassgold et al. 2004), a role that has been studied earlier in the case of active galactic nuclei (in the context of the "lamppost model", see, e.g., Nayakshin \& Kallman 2001). We speculate that protostellar jets in general develop the same kind of jet $\mathrm{X}$-ray emission, but these sources remain undetected close to the star because of strong photoelectric absorption. In those cases, jet X-rays may act as a dispersed ionization source to affect a larger volume of gas than the stellar coronal source alone.

Acknowledgements. M.A. acknowledges support from a Swiss National Science Foundation Professorship (PP002-110504). M.A. and S.S. acknowledge support by NASA through CXC award SAO GO6-7003. The CXC X-ray Observatory Center is operated by the Smithsonian Astrophysical Observatory for and on behalf of the NASA under contract NAS8-03060.

\section{References}

Anders, E., \& Grevesse, N. 1989, Geochim. Cosmochim. Acta, 53, 197

Anglada, G. 1995, Rev. Mex. Astron. Astrofis., 1, 67

Arnaud, K. A. 1996, in Astronomical Data Analysis Software and Systems V, ed. G. Jacoby, \& J. Barnes (San Francisco: ASP), ASP Conf. Ser., 101, 17

Bacciotti, F., Mundt, R., Ray, T. P., et al. 2000, ApJ, 537, L49

Bacciotti, F., Ray, T. P., Mundt, R., Eislöffel, J., \& Solf, J. 2002, ApJ, 576, 222

Bachiller, R. 1996, ARA\&A, 34, 111

Bally, J., Feigelson, E., \& Reipurth, B. 2003, ApJ, 584, 843

Blandford, R. D., \& Payne, D. G. 1982, MNRAS, 199, 883

Bouvier, J., Cabrit, S., Fernandez, M., Martín, E. L., \& Matthews, J. M. 1993, A\&A, 272, 176

Briceño, C., Luhman, K. L., Hartmann, L., Stauffer, J. R., \& Kirkpatrick, J. D. 2002, ApJ, 580, 317

Carter, C., Karovska, M., Jerius, D., Glotfelty, K., \& Beikman, S. 2003, Astronomical Data Analysis Software and Systems XII, ed. H. E. Payne, R. I. Jedrzejewski, \& R. N. Hook, 477

Cash, W. 1979, ApJ, 228, 939

Cohen, M., \& Fuller, G. A. 1985, ApJ, 296, 620

Curiel, S., Rodríguez, L. F., Moran, J. M., \& Cantó, J. 1993, ApJ, 415, 191
Dougados, C., Cabrit, S., Lavalley, C., \& Ménard, F. 2000, A\&A, 357, L61 Ducourant, C., Teixeira, R., Périé, J. P., et al. 2005, A\&A, 438, 769 Dupree, A. K., Brickhouse, N. S., Smith, G. H., \& Strader, J. 2005, ApJ, 625, L131

Dutrey, A., Guilloteau, S., Duvert, G., et al. 1996, A\&A, 309, 493

Eislöffel, J., \& Mundt, R. 1998, AJ, 115, 1554

Favata, F., Fridlund, C. V. M., Micela, G., Sciortino, S., \& Kaas, A. A. 2002, A\&A, 386, 204

Garcia, P. J. V., Ferreira, J., Cabrit, S., \& Binette, L. 2001a, A\&A, 377, 589

Garcia, P. J. V., Cabrit, S. M., Ferreira, J., \& Binette, L. 2001b, A\&A, 377, 609 Glassgold, A. E., Najita, J., \& Igea, J. 2004, ApJ, 615, 972

Grosso, N., Feigelson, E. D., Getman, K. V., et al. 2006, A\&A, 448, L29

Güdel, M., Skinner, S. L., Briggs, K. R., et al. 2005, ApJ, 626, L53

Güdel, M., Briggs, K. R., Arzner, K., et al. 2007a, A\&A, 468, 343

Güdel, M., Telleschi, A., Audard, M., et al. 2007b, A\&A, 468, 515

Hamann, F. 1994, ApJS, 93, 485

Hartigan, P., Edwards, S., \& Ghandour, L. 1995, ApJ, 452, 736

Johns-Krull, C. J., \& Herczeg, G. J. 2007, ApJ, 655, 345

Hirth, G. A., Mundt, R., \& Solf, J. 1997, A\&AS, 126, 437

Kitamura, Y., Kawabe, R., \& Saito, M. 1996a, ApJ, 457, 277

Kitamura, Y., Kawabe, R., \& Saito, M. 1996b, ApJ, 465, L137

Königl, A., \& Pudritz, R. E. 2000, Protostars and Planets IV (Tucson: University of Arizona Press), 759

Kwan, J., Edwards, S., \& Fischer, W. 2007, ApJ, 657, 897

Lavalley, C., Cabrit, S., Dougados, C., Ferruit, P., \& Bacon, R. 1997, A\&A, 327, 671

Lavalley-Fouquet, C., Cabrit, S., \& Dougados, C. 2000, A\&A, 356, L41

Leinert, C., Haas, M., Mundt, R., Richichi, A., \& Zinnecker, H. 1991, A\&A, 250,407

Muzerolle, J., Hartmann, L., \& Calvet, N., 1998, AJ, 116, 2965

Nayakshin, S., \& Kallman, T. R. 2001, ApJ, 546, 406

Pravdo, S. H., \& Tsuboi, Y. 2005, ApJ, 626, 272

Pravdo, S. H., Feigelson, E. D., Garmire, G., et al. 2001, Nature, 413, 708

Pravdo, S. H., Tsuboi, Y., \& Maeda, Y. 2004, ApJ, 605, 259

Preibisch, T., Kim, Y.-C., Favata, F., et al. 2005, ApJS, 160, 401

Pyo, T.-S., Kobayashi, N., Hayashi, M., et al. 2003, ApJ, 590, 340

Raga, A. C., Noriega-Crespo, A., \& Velázquez, P. 2002, ApJ, 576, L149

Ray, T. P., Muxlow, T. W. B., Axon, D. J., et al. 1997, Nature, 385, 415

Reipurth, B., \& Bally, J. 2001, ARA\&A, 39, 403

Rodríguez, L. F. 1995, Rev. Mex. Astron. Astrofis., 1, 10

Safier, P. N. 1992, ApJ, 392, 492

Shu, F. H., Najita, J. R., Shang, H., \& Li, Z.-Y. 2000, Protostars and Planets IV (Tucson: University of Arizona Press), 789

Solf, J., \& Böhm, K. H. 1993, ApJ, 430, L31

Stapelfeldt, K., Burrows, C. J., Krist, J. E., \& the WFPC2 Science Team. 1997, in Herbig-Haro Flows and the Birth of Stars, ed. B. Reipurth, \& C. Bertout (Dordrecht: Kluwer), IAU Symp., 182, 355

Takami, M., Chrysostomou, A., Bailey, J., et al. 2002, ApJ, 568, L53

Telleschi, A., Güdel, M., Briggs, K. R., Audard, M., \& Scelsi, L. 2007a, A\&A, 468,443

Telleschi, A., Güdel, M., Briggs, K. R., Audard, M., \& Palla, F. 2007b, A\&A, 468,425

Testi, L., Bacciotti, F., Sargent, A. I., Ray, T. P., \& Eislöffel, J. 2002, A\&A, 349, L31

Tsujimoto, M., Koyama, K., Kobayashi, N., et al. 2004, PASJ, 56, 341

Uchida, Y., \& Shibata, K. 1985, PSAJ, 37, 515

Vuong, M. H., Montmerle, T., Grosso, N., et al. 2003, A\&A, 408, 581

White, R. J., \& Ghez, A. M. 2001, ApJ, 556, 265

White, R. J., \& Hillenbrand, L. A. 2004, ApJ, 616, 998 